\title{
Inharmonicity of nonuniform overwound strings
}

\author{
P. CHUMNANTAS, C.A. GREATED and R. PARKS \\ Department of Physics, University of Edinburgh, Edinburgh EH9 3JZ, U.K.
}

\begin{abstract}
In our previous paper [10], the transverse wave equation for the case of a stepped stiff string was described, and the numerical results of the frequency equation were compared with data from experiments on piano strings. It was evident from these results that the theory presented there gave a better fit to measured inharmonicities than Fletcher's analysis for a uniform overwound string. In this paper, we describe measurements of inharmonicity carried out on nonuniform overwound strings mounted on a rigid monochord. The measurements are in good agreement with the theoretical predictions taking account the nonuniformity of the winding.
\end{abstract}

\section{INTRODUCTION}

[1-8] The natural frequencies of a piano string depart somewhat from the harmonic series; the departure is called inharmonicity. In the late 19 th century, Lord Rayleigh ${ }^{[2]}$ described a theory for the vibration of strings, showing that in the piano, the stiffness of the strings affects the restoring force to a significant degree. He derived a formula to predict how the stiffness of a piano string can cause it to vibrate at frequencies somewhat greater than those of the ideal string. In 1964, Fletcher ${ }^{[8]}$ conducted a more accurate treatment of Rayleigh's method, considering both clamped and hinged boundary conditions. He found that the equation was of the form $f_{n}=n F\left(1+B n^{2}\right)^{1 / 2}$, where $F$ and $B$ are constants. $B$ is described as the inharmonicity coefficient. Actually, all piano bass strings are characterised by a steel wire core wrapped with copper, or sometimes iron, used to increase the string's linear mass density. While the tight coiling of copper wire ensures close coupling to the core, the windings contribute considerably more to the increase in the string's linear mass density than to its bending stiffness. Most bass strings have a single winding of copper wire, and it is usually only within the lowest octave that double winding is used. A double-wound string consists of a bare steel core wrapped with a small diameter copper wire, which is then overspun with a second winding of larger diameter. A small part of the steel core is left exposed near the end of the string. The inharmonicity of the overwound piano strings does not only depend on the string stiffness; since the copper windings do not extend up to the string's supports, the resulting nonuniformity in the linear mass density must also be taken into account. 
In a previous paper [10], an expression for the frequencies of vibration of a stepped stiff string was described. In the present paper this expression is used to calculate the inharmonicity of piano string with nonuniform winding. The theoretical results are compared with measurements carried out on a rigid monochord.

\section{THEORETICAL CONSIDERATION AND NUMERICAL RESULTS}

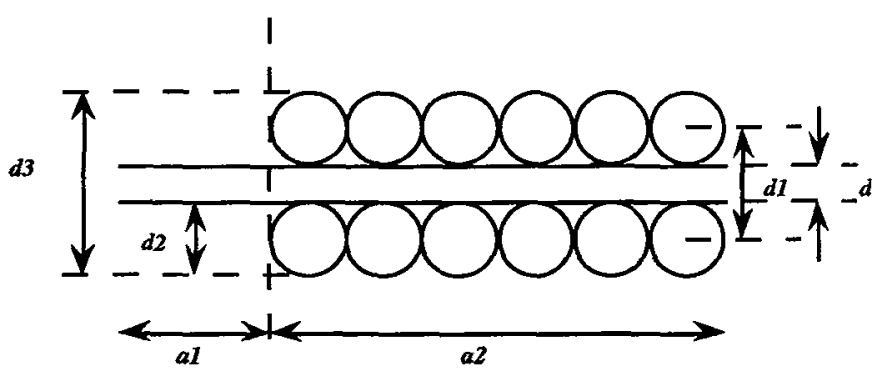

Fig. I The 2-segment overwound string.

Consider the frequency equation [10] in the case of the 2 segment stiff string (see Fig. I). Its frequency equation is

$$
\begin{aligned}
& \left(\frac{\mu_{11}^{2}}{\mu_{22}^{2}}+1\right)\left(\frac{\mu_{12}^{2}}{\mu_{21}^{2}}+1\right)\left\{\frac{\mu_{11}}{\mu_{21}} \frac{\tanh \left(\mu_{21} a_{2}\right)}{\tanh \left(\mu_{11} a_{1}\right)}+1\right\}\left\{\frac{\mu_{12}}{\mu_{22}} \frac{\tan \left(\mu_{22} a_{2}\right)}{\tan \left(\mu_{12} a_{1}\right)}+1\right\} \\
& -\left(\frac{\mu_{11}^{2}}{\mu_{21}^{2}}-1\right)\left(\frac{\mu_{12}^{2}}{\mu_{22}^{2}}-1\right)\left\{\frac{\mu_{11}}{\mu_{22}} \frac{\tan \left(\mu_{22} a_{2}\right)}{\tanh \left(\mu_{11} a_{1}\right)}+1\right\}\left\{\frac{\mu_{12}}{\mu_{21}} \frac{\tanh \left(\mu_{21} a_{2}\right)}{\tan \left(\mu_{12} a_{1}\right)}+1\right\}=0 \\
& \mu_{j k}=\left\{\sqrt{\left(\frac{T_{j}}{2\left(Q S \kappa^{2}\right)_{j}}\right)^{2}+(2 \pi f)^{2} \frac{\rho_{j}}{\left(Q \kappa^{2}\right)_{j}}}+(-1)^{k} \frac{T_{j}}{2\left(Q S \kappa^{2}\right)_{j}}\right)^{\frac{1}{2}}
\end{aligned}
$$$$
: j, k=1,2 \text {. }
$$

where $\mathrm{T}$ is the tension, $\mathrm{S}_{\mathrm{j}}$ is the area of cross-section and $\kappa_{j}$ the radius of gyration. $\rho_{j}$ and $Q_{j}$ are the density and modulus of elasticity of the material for the section $j$ of the string.

The allowed frequencies, $f_{n}:(n=1,2,3,4, \ldots)$ can be found from equations (1) \& (2). The values were found numerically by applying Newton's method; this was programmed on an Apple Macintosh computer using the Mathematica package. Numerical calculations have been undertaken to compute theoretical mode frequencies for several 2 segment overwound strings. Fig. I shows the notation used for defining the parameters of the overwound string. This was clamped at both ends and the linear density was calculated using the method of W T Goddard [9]. The first segment is the bare string $\left(a_{1}\right)$ and the second segment has both the steel core and the wrapped copper wire $\left(a_{2}\right)$. Graph I presents the relation between the square of the theoretical mode frequency per the square of mode number $\left(f_{n} / n\right)^{2}$ and the square of the mode number $\left(n^{2}\right)$, for a uniform overwound string (length $1=80.0 \mathrm{~cm}$.) and the 2 segment overwound string $\left(a_{1}=5.0 \mathrm{~cm}\right.$. and $a_{2}=75.0 \mathrm{~cm}$.). It shows that the relation is linear in the case of the uniform overwound string. This is the case dealt with by Fletcher, and our results agree. In the case of the stepped overwound string, the inharmonicity is substantially increased, and the line is evidently curved. Graph II displays the residuals (the deviations from the best straight line fit to the data) for three 2-segment overwound strings. These have the same diameters $\left(\mathrm{d}_{1}=1.35 \mathrm{~mm}\right.$. and $\mathrm{d}_{2}=4.20 \mathrm{~mm}$.), and the same lengths of bare steel string $\left(\mathrm{a}_{1}=5.0 \mathrm{~cm}\right.$.), but their overwound lengths are $\mathrm{a}_{2}=75.0,100.0$ and $125.0 \mathrm{~cm}$., respectively. 


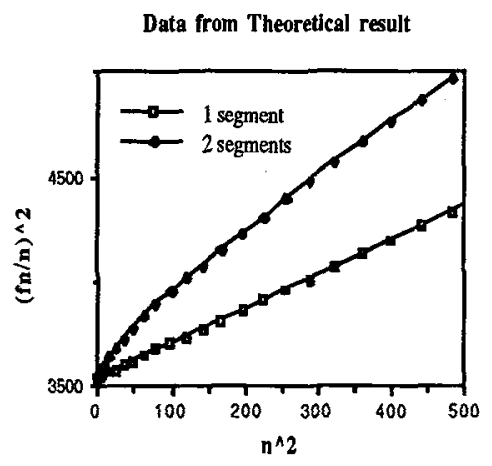

Graph I: The relation between the square of theoretical mode frequencies per the square of mode number $\left(\mathrm{f}_{\mathrm{n}} / \mathrm{n}\right)^{2}$ and the square of mode number $\left(n^{2}\right)$ for a uniform overwound string with $I=80.0 \mathrm{~cm}$. and a 2 segment overwound string with $\mathrm{a}_{1}=5.0 \mathrm{~cm}$. and $\mathrm{a}_{2}=75.0 \mathrm{~cm}$.

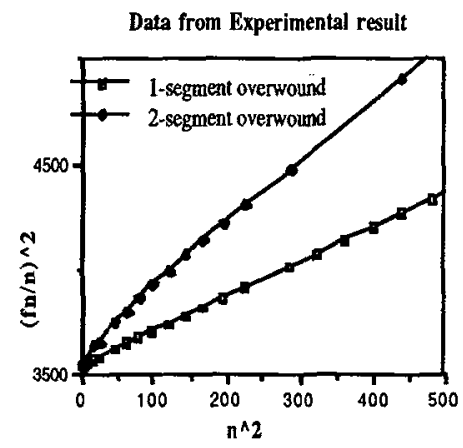

Graph III: The relation between the square of experimental mode frequencies per the square of mode number $\left(f_{n} / n\right)^{2}$ and the square of mode number $\left(n^{2}\right)$ for a uniform overwound string with $I=80.0 \mathrm{~cm}$. and a 2 segment overwound string with $\mathrm{a}_{1}=5.0 \mathrm{~cm}$. and $\mathrm{a}_{2}=75.0 \mathrm{~cm}$.

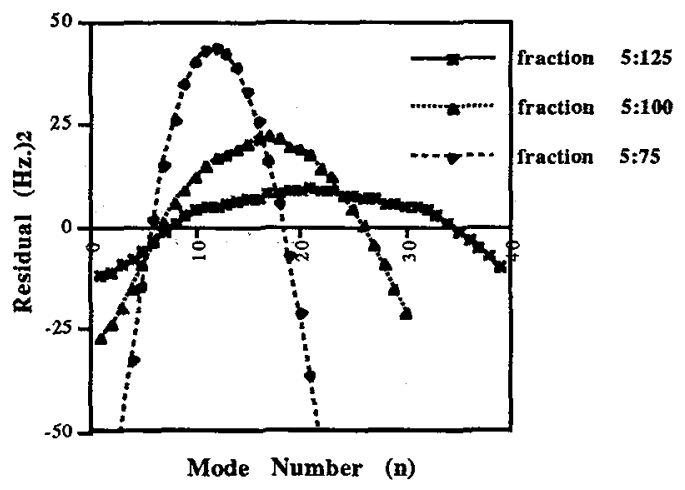

Graph II: The residuals from a straight line of the theoretical resuits for three 2segment overwound strings with the same diameters $\left(d_{1}\right.$ and $\left.d_{2}\right)$, and the same length of bare string $\left(a_{1}=5.0 \mathrm{~cm}\right.$.), but with $a_{2}=75.0$, 100.0 and $125.0 \mathrm{~cm}$.

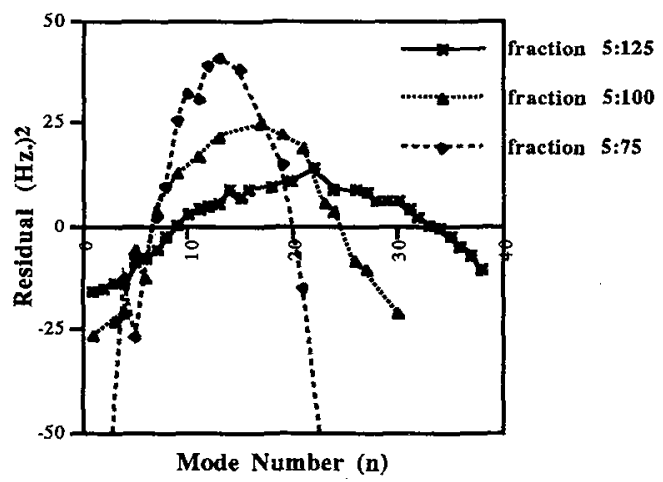

Graph IV The residuals from a straight line of the experimental results for three 2 segment overwound strings with the same diameters $\left(d_{1}\right.$ and $\left.d_{2}\right)$, and the same length of bare string $\left(a_{1}=5.0 \mathrm{~cm}\right.$.), but with $a_{2}=75.0$, 100.0 and $125.0 \mathrm{~cm}$. 


\section{EXPERIMENTAL RESULTS}

The experiment studied the vibration of 2 segment overwound strings (see Fig. I) on a purposed-designed monochord. The monochord is composed of a rigid steel bar, 2 pairs of specially designed rigid clamps, 2 tuner supports, and 2 tuners. Each pair of clamps stops each end of the string at 3 points in a plane perpendicular to the string length. The strings were plucked at a position close to the end and the sound was recorded at a point near to the centre of the string using a microphone mounted a short distance above. The acoustic signal was captured digitally using an A/D converter and was analysed using an FFT. A program developed in Edinburgh [11] locates the peaks in the spectrum with high accuracy.

The relation between the square of experimental mode frequencies per the square of mode number $\left(f_{n} / n\right)^{2}$ and the square of mode number $\left(n^{2}\right)$ for a uniform overwound string (length $1=$ $80.0 \mathrm{~cm}$.) and for a 2 segment overwound string $\left(\mathrm{a}_{1}=5.0 \mathrm{~cm}\right.$. and $\mathrm{a}_{2}=75.0 \mathrm{~cm}$.) are shown in Graph III. The relation is not linear in the case of the nonuniform overwound string, in agreement with the theoretical result. Experimental results for three 2 segment overwound strings are shown in Graph IV as residuals from a straight line. It is seen that the greater the fraction $\left(a_{1} / a_{2}\right)$, the more curving is the trend. The theoretical result in Graph II gives a very good match.

\section{CONCLUSION}

The results from theory and experiment presented above show that the mode frequencies of nonuniform overwound strings depart from those predicted by Fletcher's equation. It is evident that this departure, graphed as the residual from a straight line fit to Fletcher's equation, gives a curving trend whose significance depends on the fraction $a_{1}: a_{2}$. The greater the fraction $a_{1}: a_{2}$, the more curving is the trend. For normal bass piano strings, the fraction of unwound string is usually considerably less than 1:25. Thus a straight line fit to Fletcher's equation is a resonable approximation. However, the inharmonicity coefficient will be considerably increased by the nonuniformity of the winding.

\section{REFERENCES}

[1] D.M. Campbell, C.A. Greated and R. Parks, Proc. of the Inst. of Acoustics, 12(1), 743-752 (1990).

[2] J.W. Strutt Lord Rayleigh, "The Theory of Sound", 2nd ed. Dover Publications, New-York, 1954.

[3] P.M. Morse, "Vibration and Sound", 2nd ed. McGraw-Hill, New York, 1948.

[4] R.S. Shankland and J.W. Coltman, J. Acoust. Soc. Amer., 10(3), 161-1666 (1939).

[5] O.H. Shuck \& R.W. Young, J. Acoust. Soc. Amer., 15(1), 1-11 (1943).

[6] R.W. Young, J. Acoust. Soc. Amer., 24(3), 267-273 (1952).

[7] R.W. Young, Acustica, 4, 259-262 (1954).

[8] H. Fletcher, J. Acoust. Soc. Amer., 36(1), 203-210 (1964).

[9] W. Trow Goddard, "A handbook on the strings of the pianoforte and other keyboard instruments with design formulae", Billingham Press, Billingham, England, 1985.

[10] P Chumnantas, C A Greated and D M Campbell, Proc. of the Inst. of Acoustics, 15(3), 665-672 (1993).

[11] R Parks, Proc. of the Inst. of Acoustics, 15(3), 673-680, (1993). 